\title{
Development and use of a back to work symptom and risk assessment tool to reduce COVID-19 disease exposure
}

\author{
Benjamin Schooley \\ University of South Carolina \\ bschooley@,cec.sc.edu
}

\author{
Sue S. Feldman \\ University of Alabama Birmingham \\ sfeldman@uab.edu
}

\begin{abstract}
Technology solutions to mitigate repercussions of the COVID-19 pandemic include tools that provide guidelines and interfaces to guide behavior, reduce exposure to the disease, and enable policy-driven avenues to return to a sense of normalcy (e.g., work and school). This paper takes a design science approach to present the justification, design, development, and early assessment of a return-towork COVID-19 symptom checker and risk assessor. The system was implemented across 34 institutions of health and education in the US State of Alabama, including over $174 k$ users with $>4$ million total uses and $>86 k$ reports of exposure risk between July 2020 and April 2021. Users complied with use policies between 60-74\% of the time, with $k-12$ schools showing higher compliance than colleges and universities. Using system use data and focus group discussions, findings indicate the system was generally accepted, used regularly, facilitated reduction of disease exposure, and enabled a path back to work and school.
\end{abstract}

\section{Introduction}

By the end of May 2021, there had been over 33 million cases of COVID-19 and close to 600,000 deaths in the US and 32 million cases and over 700,000 deaths in the EU/EEA and almost 7 million deaths worldwide. The COVID-19 pandemic has caused economic and social turmoil to tens of millions of people worldwide, revealing vulnerability in our healthcare systems and increased reliance on technology-enabled applications and electronic tools.

The pandemic has changed the way we socialize, work, learn, and act around others. The pandemic caused several changes to come into effect at a rapid pace. Telework, telehealth, and online education became essential in helping society mitigate infection exposure and spread while still caring for its most crucial needs. Many electronic tools have been released and used for patients and healthcare providers alike. Indeed, remote care and monitoring have become an important and often critical component of our healthcare delivery system.
The push for disease mitigation has led to innovative technologies and creative ways of coping with the damage caused by COVID-19. The quick transition to online life and the rapid demand for certain services to remain available revealed opportunities for developing new technologies, or repurposing current technologies, that could impact how the public interfaces with COVID-19 in and around proximal and more distal social and professional circles.

Technology solutions to mitigate repercussions of the pandemic have been focused on three components that were initially used in controlling and managing chronic diseases in the data-people-system framework by Bardhan, Chen, and Karahanna [1] (1) collection, derivation, integration, and articulation of health data; (2) interoperability of systems; and (3) guidelines and interfaces to guide people's behavior. The support of technology experts worldwide revealed solutions that provided population screening, tracking infections, prioritizing the use and allocation of resources, and designing targeted responses.

With the use of technology-enabled applications and electronic tools such as QR codes, symptoms checkers, chatbots, and telehealth software, the general hope has been that the spread of COVID-19 has been mitigated. However, little is known about the effectiveness and impacts of these tools. This research investigates a statewide initiative in Alabama where a COVID assessment tool was implemented across K12 and higher education institutions. Students, staff, and faculty were provided a tool to self-assess COVID-19 symptoms that calculated a risk level with actional information, and then presented the user with a green or red passport for entering campus. The key question assessed in this research is the association between the tool and exposure to COVID-19. We explore the design, development, and use of the GuideSafe $^{\text {TM }}$ Healthcheck app as an effective method to reduce disease exposure in Alabama. 


\section{Background}

\subsection{COVID-19 Impact and Symptom Checking}

The Centers for Disease Control and Prevention (CDC) and the World Health Organization (WHO) have issued weekly COVID data tracking and epidemiological reports to summarize cases and deaths, vaccine uptake, virus variants, and other information pertinent to the pandemic situation. At the end of May 2021, the CDC reported a drop in US COVID-19 cases and deaths to the lowest levels in nearly a year. During the same period, the WHO reports there have been significant increases in case and death instances in regions such as the Western Pacific and Africa, despite a decrease in global COVID-19 cases and deaths. While the extent of the pandemic has ebbed and flowed in different locations and at different times, the CDC reports that COVID19 and its's variants will be a challenge for years to come. Hence the need for continued vigilance in combating its spread.

While education and awareness are critical, reinforcement in the form of symptom assessment tools provide an avenue for regular reminders, and personal assurance about risk levels post screening. Screening tools can also serve to increase symptom awareness and behavior change, especially as symptoms change over time. Most COVID symptom lists include the constellation of fever or chills, cough, shortness of breath or difficulty breathing, fatigue, muscle or body aches, headache, new loss of taste or smell, sore throat, congestion or runny nose, nausea or vomiting, and diarrhea. ${ }^{1}$ Symptom assessment tools help the patient assess and, with the help of built-in guidance, to self-triage. If the user obtains a result which could indicate a COVID-19 infection, some tools may provide actionable guidance specific to the user and the symptoms indicated.

Symptom checking apps do pose challenges, including the correctness of a symptom assessment and privacy of user information. For the former issue, it was found that most symptom assessment tools differ in their correct assessment of COVID-19 and control cases and that a balance between usability and clinical specificity is needed. For the latter issue, many citizens may feel symptom assessment (whether inperson or as an app) is a violation of privacy [2]. Governments or other institutions may mandate use of such a tool and have done so in various settings across the world. For example, in India, the home ministry

\footnotetext{
${ }^{1}$ https://www.cdc.gov/coronavirus/
}

mandated that all workers, public or private, use a government-backed COVID-19 tracking app called Aarogya Setu. Even when government institutions do not require such technologies, employers and organizations might require them, leaving workers feeling like their privacy has been breached $[2,3]$.

\subsection{Guidelines and interfaces to guide people's behavior}

Returning to the workplace and re-starting events post pandemic lock-down meant that certain checkpoints needed to be put in place to mitigate the spread of infection [4]. As per public health guidance, employees, trainees, students, and all visitors would be required to answer a set of questions to gain access to work and school locations. Such measures would be combined with temperature checks and the use of hand sanitizer upon entering an establishment $[4,5]$.

Many organizations used COVID-19 symptom assessment tools or surveys, some with "passports" to display the results of the symptom assessment tool. These surveys require the user to review a list of symptoms and potential exposure questions. The surveys might be available as a web-based or mobilebased app or as a physical document. Requirements generally include daily completion before entering the place of work, study, or care center. The employer or administrator is then informed of the completed survey through a compliance report. The person would not be allowed to enter the premises unless the survey, which may be particular to each workplace, is conducted and then yields a green passport, indicating entry. Typically, the green passport would be displayed for ready visualization by anyone checking.

In the education environment, many schools and universities developed a variety of surveys. Most of these were confined to that environment, in other words developed by that school for use by that school. The CDC's Coronavirus Self-Checker is available on their website and also as a customizable widget that health departments and healthcare systems could add to their website. Other companies have also built apps that can be used for screening, tracking, and providing notifications for K-12 schools.

\subsection{Limitations of the back-to-work passport}

Silent spreaders either as pre-symptomatic or asymptomatic infected individuals cannot be detected by apps or questionnaires that conduct health checks. Symptoms may take from 2 to 14 days to develop after exposure to the virus (cdc.gov/coronavirus). Even 
early symptoms can sometimes be dismissed as tiredness by a busy parent or an overworked student. For example, those suffering with seasonal allergies may dismiss a slight cough as hay fever; asthmatics may mask symptoms by using an inhaler.

On the other hand, since many symptoms of COVID-19 are present in other illnesses, including chronic medical conditions, some individuals may be repeatedly excluded from work or school even though they do not have COVID-19 (or any other contagious illness). This is problematic as these students or workers may also frequently miss school/work due to their medical conditions.

In-person checks require more staff and a dedicated space in order to be conducted safely. Nonmedical staff may not have enough knowledge of the individuals being assessed to make decisions when it comes to symptoms occurring in chronic conditions, or assessing the likelihood that the person was exposed to the virus. The volunteers or school personnel are required to be in frequent and close contact with a large number of persons, which could increase the risk of COVID-19 exposure for all parties involved, especially if logistics do not allow for a dedicated space that is large enough for the activity to be conducted safely.

Due to these limitations, the CDC does not currently recommend routine in-person school-based COVID-19 symptom screening of all students, and it encourages home symptom screening. Additionally, waiting until someone is on campus to check symptoms increases the risk of exposure, whereas home screening in the presence of a risk stratification and passport deceases exposure.

One must also take into consideration perceived or real fears from consequences of missing work or reluctance to stay at home due to abusive or stressful situations [6]. One of the key issues is when people do not have enough sick time to stay home for the 2 -week requirement.

The availability of a multitude of apps for information, symptom assessment, and contact tracing has increased the time spent online and relying on the internet and social media for information, the risk of harm from misinformation, malware, and ransomware has increased. Emphasis should be placed on only downloading and using official apps from trusted sources. In April 2020, the European Commission published Guidance on Apps supporting the fight against COVID-19 pandemic in relation to data protection.

Reliance on digital technology can highlight socioeconomic inequalities, further contributing to healthcare disparities. To integrate such technology for use to mitigate infection spread, this type of response needs to include interventions that place most, if not all, on equal footing. While Healthcheck was developed as a web-based app accessible on any computer or mobile device, the GAEN requires a specific operating system on the smart phone, thereby eliminating swaths of populations. Interoperability of apps and availability of WiFi hotspots in all areas guides this response in a comprehensive way.

\section{Research Approach}

This research follows a design science research (DSR) methodology inclusive of (i) problem identification and motivation, (ii) definition of the objectives for a solution, (iii) design and development, (iv) demonstration, (v) evaluation, and (vi) communication $[7,8]$. This paper reports on the design of a software artifact for a COVID-19 symptom checker and return-to-work/school passport in Alabama called Healthcheck, developed at the University of Alabama at Birmingham, endorsed by the Governor of Alabama, and implemented statewide to K-12 schools, public and private and Institutions of Higher Education, public and private (www.guidesafe.org). Healthcheck was required for daily use and instrumental in keeping instruction in person and on campus in Alabama. Once the user answered the symptom assessment and exposure questions, the answers were run through an algorithm that resulted in actionable guidance and a passport for campus or event entry (such as athletic events).

The work was completely led by design science researchers from within the University setting. We organized the components in the app into design principles and features for designing a COVID-19 return-to-work passport app. We use guidance from mode $3 \mathrm{C}$ and $4 \mathrm{~B}$ of design theorizing to contribute to knowledge for a solution design process and system to inform the design of a DSR project and codify effective design principles and features that contribute to future DSR projects for similar contexts [9]. The design requirements, artifact design and development, and first phase of evaluation are described in the sections below.

\subsection{System Requirements}

Drawing from CDC guidelines for COVID-19, a set of guiding principles for a passport app were determined using a DELPHI method $[10,11]$ to interview and gain feedback and consensus from two lead health informatics faculty $(n=2)$, software developers $(n=2)$, and University senior administrators $(n=15)$ across functional units at a major medical university in the Southeast. The overarching goal of 
the Healthcheck application was to provide a platform for work/school re-entry for educational institutions and to serve as the cornerstone for the state of Alabama's commitment to support education across the entire state. The Healthcheck application was considered one integral component of a comprehensive work/school re-entry plan inclusive of virus testing, education and communication about safe behaviors, social distancing, symptom checking, and exposure notification as illustrated in Figure 1.

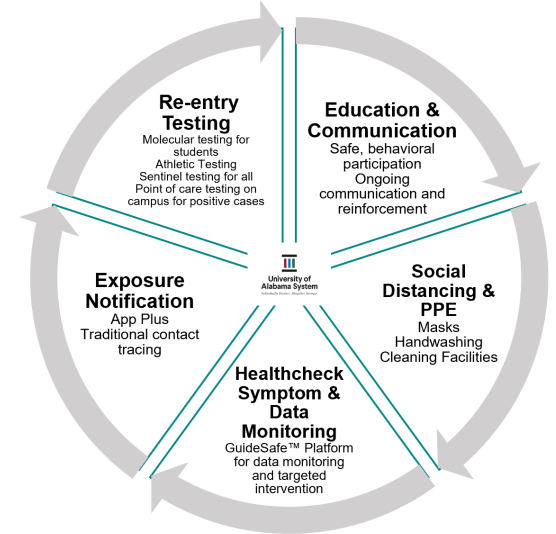

Figure 1. Comprehensive work/school reentry strategy for Alabama

The comprehensive education re-entry plan included the following:

Users: All new and returning faculty, staff and students who at any point during their job performance or education/training program must physically enter campus.

When to use: Prior to return to campus and ongoing on a designated frequency. Phased Re-entry.

Policy requirements on users:

- Required completion of COVID-19 online training module(s). Required Healthcheck use prior to return to campus and after return to campus (to be completed daily with compliance at 3 days and enforcement at 6 days

- Requiring SARS-CoV-2 testing or documentation of a negative test for all faculty, staff and students prior to return to campus and either quarantine for CDC recommended number of days or negative test result after reporting high risk symptoms using Healthcheck.

- Healthcheck - on-going symptom monitoring

To support the work/school re-entry plan, the high-level meta-requirements for the Healthcheck app include:

- The app must facilitate a strategy to manage large groups and mitigate risk to further exposure to COVID-19
- Ability to understand and track the change from the COVID-19 screening process including in test status and symptoms and facilitate self-reporting and appropriate institutional response to new infection

- Ability for people to self-identify as at-risk for greater disease burden from the COVID-19 and for the institution to facilitate safe return for those at greater risk

- Ability to potentially report exposure to COVID19 positive individuals to facilitate self-reporting of exposure in order to limit the spread of the virus

- Balance the return to normal operations with the health and safety of employees, students and visitors

- No personally identifiable information shall be exposed for any user of the system

The Healthcheck passport tool was envisioned by designers to provide a risk assessment and report for returning to work/school. The final screen of the app would thus provide an indicator for good to enter (e.g., green), or red for not good to enter, to be used for campus and sporting event entrance. Risk level calculations were determined as follows:

\section{Risk level 3 Calculation Method: (RED)}

Those users whose Healthcheck selections match the below criteria receive risk level 3:

1. user has been in isolation

2. user has been in contact with another person diagnosed or under investigation of COVID-19

3. user has one COVID-19 symptom including: fever, cough, difficulty breathing, loss of taste or smell

Risk level 2 Calculation: (YELLOW)

Those users whose Healthcheck selections match the below criteria receive risk level 2:

1. user reports two symptoms other than fever, cough, difficulty breathing, loss of taste or smell, or none

\section{Risk level 1 calculation: (GREEN)}

Those users whose Healthcheck selections include any items other than those described for Risk level 2 and 3 fall into this category.

Each passport would need to deliver individual actionable guidance specific for the school or organization to which it applied. While each school or organization could set their own minimum compliance standard, it was envisioned to set it daily, including weekends and holidays if campus attendance would be needed. For those who would be exempt from being on campus (i.e., remote workers), Healthcheck would not be required.

The digital format of the Healthcheck app would need to enable updates in a continuously changing environment, which may also mean a change in 
protocols for screening as informed by health authorities. As well as being flexible, this format would need to be a scalable option that helps organizations provide a user-centered solution to their safety needs.

After completing the Healthcheck activity, workers would need to receive a digital badge that clears them for the day. This badge would need to be displayed at access control points that notify employers. The workers that do not pass this clearing would be instructed to quarantine at home and be provided with advice on self-care, monitoring, testing, or additional care. This type of work passport would have the dual purpose of protecting everyone at the workplace as well as providing checks and care on an individual basis.

\subsection{Success Requirements}

Participants in the requirements, design, and development phases of the Healthcheck application noted several factors that would indicate system success and would lead to positive public health outcomes including reducing the number of people infected with COVID-19 and slowing its spread. These success factors include:

- A wide range of users will see value in using the system

- Employees and students will uptake and use the system

- Users will comply with policies to use the Healthcheck app as prescribed without missing more than three to six consecutive days between uses

- Compliance with use policies will increase over time as behavior is reinforces via other mechanisms (e.g., education, regular notifications to user via text messages, peer influence)

These success factors, driven by system stakeholders, are reviewed in the evaluation section of this manuscript.

\subsection{Design and Development}

The Healthcheck app was developed in multiple iterations using CDC guidance and input from faculty $(n=2)$, software developers $(n=2)$, University senior administrators $(n=15)$ across functional units at a major medical university, 1 of which is a public health expert, 1 infectious disease expert, and 5 MD's. This was the same group that participated in requirements determination. A 2-week pilot consisting of approximately 200 research staff was conducted at the University of Alabama Birmingham in May 2020. Voluntary feedback was provided by pilot participants throughout the two weeks and issues were prioritized by the research team for inclusion in the next app version. Based on user feedback, the app was improved for its performance, usability, and security.

The app was designed to include 5 primary screens as illustrated in Figures 2-6. The first screen (Figure 2) is the login screen that authenticates with an organization's central authentication system. User's are required to use the app daily prior to attending work/school.
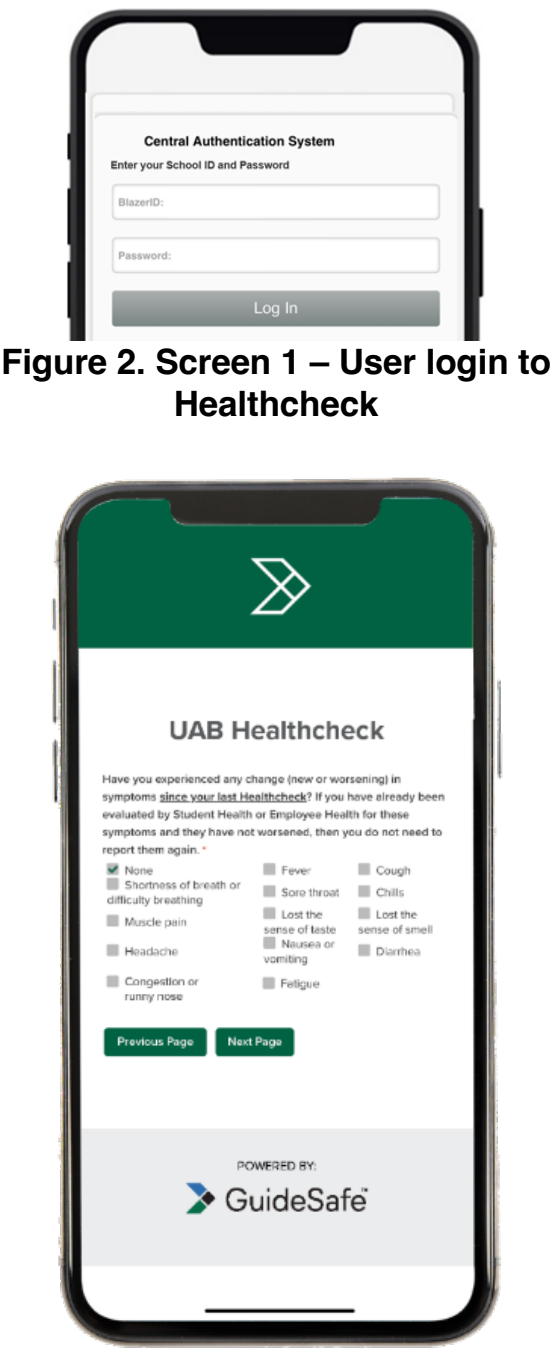

Figure 3. Screen 2 - COVID-19 Symptom assessment 


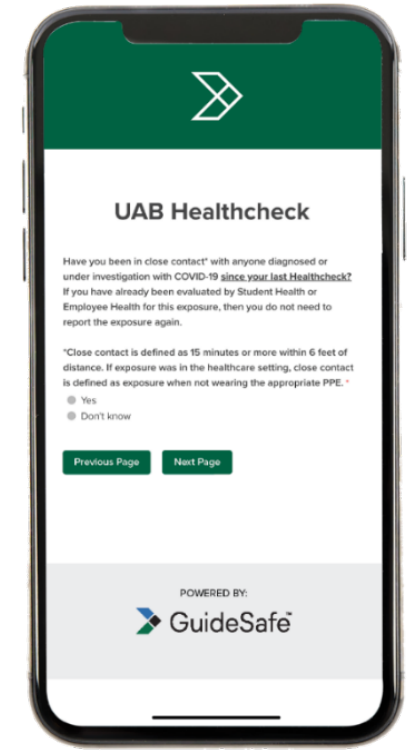

Figure 4. Screen 3 - COVID-19 exposure assessment

The second screen (Figure 3) checks for COVID19 symptoms as per CDC guidelines, while the screen illustrated in Figure 4 asks the user to report if he/she has been in contact with another person who was diagnosed or under investigation of COVID-19 since the users last Healthcheck report.

The next screen (Figure 5) asks whether the user had been quarantined or recommended to be in quarantine. Figure 6 displays the user's risk level. If the user shows low risk, he/she is able to attend work/school subject to any other additional screenings or healthcare provider recommendations.

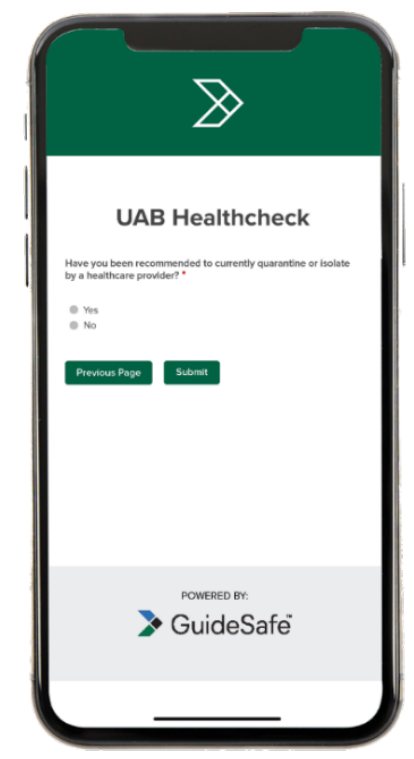

\section{Figure 5. Screen 4 - COVID-19 quarantine} assessment

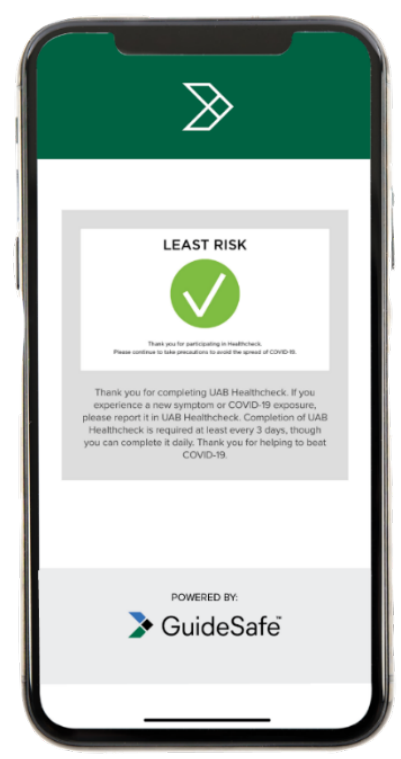

Figure 6. Screen 5 - COVID-19 risk level reporter

All of the above requirements were validated by the participants in the requirements phase prior to moving into the design and development phase of work.

\section{Evaluation}

Artifact evaluation focused on addressing success requirements (see section 3.2). System use data was used to assess user uptake, continued use over time, and functionality used (i.e., symptoms reported, quarantine reported, vaccination reported, risk level assessments), and compliance with use policies (i.e., not miss more than 3-6 days between uses). Qualitative focus groups were held to assess perspectives about the Healthcheck app, including from diverse user groups. These are described below.

\subsection{System Use}

The Healthcheck app was implemented across the state of Alabama to K-12 and institutions of higher education starting in May 2020 with organizational sign-up and training and continues to be used at the time of this manuscript submission in June 2021, although this paper reports on the period from July 3 , 2020 through April 30, 2021. It was expected that the system would be acquired by educational institutions from across the state and that it would be used regularly by users. A total of 34 organizations in the 
state of Alabama participated spanning 10 independent K-12 schools, 2 K-12 school districts, 1 government agency, 9 small colleges $(<3 \mathrm{~K}$ student enrollment), 8 mid-sized colleges and universities (between $4 \mathrm{k}$ to $10 \mathrm{k}$ student enrollment), and 5 large universities $(>14 \mathrm{k}$ student enrollment). These organizations accounted for over $174 \mathrm{k}$ unique users and 4.8 million total daily entries into the Healthcheck system (see Tables 1-4).

\begin{tabular}{|l|l|l|}
\hline & Unique Users & Total Entries \\
\hline K12 & 1,440 & 60,097 \\
\hline $\begin{array}{l}\text { Government } \\
\text { Agency }\end{array}$ & 221 & 7,406 \\
\hline Small School & 6,913 & 233,151 \\
\hline $\begin{array}{l}\text { Medium } \\
\text { School }\end{array}$ & 34,842 & 897,381 \\
\hline Large School & 130,909 & $3,614,767$ \\
\hline Total & 174,325 & $4,812,802$ \\
\hline
\end{tabular}

Table 1. Total users and system entries

High risk for COVID-19 was self-reported 86,470 times by $37,420(21.5 \%)$ individuals during the study period. Risk level 2 was never reported during the study period, leaving all remaining reports at risk level 1 or 0 (see Tables 2-3).

\begin{tabular}{|l|l|l|}
\hline & $\begin{array}{l}\text { Risk } \\
\text { Level 1 }\end{array}$ & $\begin{array}{l}\text { Risk } \\
\text { Level 3 }\end{array}$ \\
\hline K12 & 59,628 & 469 \\
\hline $\begin{array}{l}\text { Government } \\
\text { Agency }\end{array}$ & 7,343 & 63 \\
\hline Small School & 229,986 & 3,165 \\
\hline Medium School & 883,009 & 14,372 \\
\hline Large School & $3,546,366$ & 68,401 \\
\hline Total & $4,726,332$ & 86,470 \\
\hline
\end{tabular}

Table 2. Total number of times risk level was reported by organization type

Reporting of symptoms, quarantine, and vaccination status was also performed by users (see Tables 4 and 5). The public health belief was that identifying COVID cases and subsequent quarantine provided much needed help to reducing disease exposure and spread of the virus and that the Healthcheck system was instrumental for doing so.

\begin{tabular}{|l|l|l|}
\hline & $\begin{array}{l}\text { Risk } \\
\text { Level 1 }\end{array}$ & $\begin{array}{l}\text { Risk } \\
\text { Level 3 }\end{array}$ \\
\hline K12 & 1,434 & 207 \\
\hline
\end{tabular}

\begin{tabular}{|l|l|l|}
\hline Gov Agency & 219 & 39 \\
\hline Small School & 6,867 & 1,134 \\
\hline Medium School & 34,409 & 6,729 \\
\hline Large School & 129,977 & 29,311 \\
\hline Total & 172,906 & 37,420 \\
\hline
\end{tabular}

Table 3. Unique users reporting risk level by organization type

\begin{tabular}{|l|l|l|l|}
\hline & Median & Min & Max \\
\hline K12 & 34 & 1 & 268 \\
\hline Gov Agency & 27 & 1 & 95 \\
\hline Small School & 12 & 1 & 268 \\
\hline Medium School & 10 & 1 & 277 \\
\hline Large School & 11 & 1 & 296 \\
\hline
\end{tabular}

Table 4. Median Number of Uses per User

\begin{tabular}{|l|l|}
\hline Jul 20 & $\begin{array}{l}\text { Quarantine by } \\
\text { month }(\mathrm{n})\end{array}$ \\
\hline Aug 20 & 4,578 \\
\hline Sept 20 & 7,227 \\
\hline Oct 20 & 5,877 \\
\hline Nov 20 & 6,710 \\
\hline Dec 20 & 6,043 \\
\hline Jan 21 & 7,486 \\
\hline Feb 21 & 2,920 \\
\hline Mar 21 & 1,037 \\
\hline Apr 21 & 807 \\
\hline Total & 42,685 \\
\hline
\end{tabular}

Table 5. Total number of times quarantine reported by month

\subsection{System Compliance}

Users were asked to use the Healthcheck app on a daily basis, to not miss more than three days between uses, and six days at a maximum. Users received notifications when they became out of compliance. That is, on day 3, they received an email message reminder. If unresponsive, they then received a text message notification on day 6 . If still unresponsive, then this same process repeated itself. If still unresponsive, then they were not sent any further reminders. This was to accommodate people on leave, vacation, or no longer with the school. In terms of 
compliance, there were a total of $4,812,802$ total events (system submissions) between July 3, 2020 and April 30, 2021, with 1,817,873 (43.6\%) within one day of each other, and 3,112,253 (74.81\%) within the prescribed three day compliance window (see Table 6). A total of $1,700,549(25.19 \%)$ events took place outside the prescribed compliance window. There were a total of $843,581(17.52 \%)$ reports that took place six or more days between entries. Seventy percent of users were out of three-day compliance at least once, with an average of 10.8 times over the study time period (see Table 6).

It was recognized that completing Healthcheck represented a behavior change and a significant change in routine. Therefore, while compliance was enforced, there was some tolerance for noncompliance. As such, results also found an improvement in 3-day compliance rates, from $62 \%$ in July 2020 to $71 \%$ in April 2021 (see Table 6), the highest months being in September - November 2020 at the height of the pandemic in Alabama. School was not in session from Nov 17 - Jan 8, and thus consistent use and tracking was not required.

\begin{tabular}{|l|c|c|r|}
\hline & $\begin{array}{l}<=3 \\
\text { days } \\
\text { from last } \\
\text { entry }\end{array}$ & $\begin{array}{l}\text { Total } \\
\text { Entries }\end{array}$ & $\begin{array}{l}\text { \% } \\
\text { Compliance }\end{array}$ \\
\hline July & 51327 & 82221 & $62.43 \%$ \\
\hline Aug & 486036 & 701164 & $69.32 \%$ \\
\hline Sept & 622443 & 827643 & $75.21 \%$ \\
\hline Oct & 522363 & 698374 & $74.80 \%$ \\
\hline Nov 1-17 & 228562 & 314148 & $72.76 \%$ \\
\hline Jan 8-30 & 260195 & 373979 & $69.57 \%$ \\
\hline Feb & 330168 & 466532 & $70.77 \%$ \\
\hline Mar & 323859 & 458938 & $70.57 \%$ \\
\hline Apr & 293972 & 415308 & $70.78 \%$ \\
\hline
\end{tabular}

\section{Table 6. 3-Day Compliance with Healthcheck use}

While many factors play into policy compliance, it was found that the Healthcheck application facilitated widescale compliance. It's interesting to note that compliance differed across organizational types, with median use and compliance being highest with K-12 institutions and lowest at large Universities.

Once vaccinations were available, a vaccination question was added to Healthcheck. Table 7 represents the data from this question. It is important to note that while all other questions were required, answering the vaccination question was not required.

\begin{tabular}{|c|c|}
\hline & $\begin{array}{c}\mathrm{N}=174,226 \\
\mathrm{n}(\%)\end{array}$ \\
\hline Yes & $33,440(19.19)$ \\
\hline Not Yet But Plan To & $123,832(71.08)$ \\
\hline Prefer Not To Answer & $16,954(9.73)$ \\
\hline
\end{tabular}

Table 7. Reported Vaccination status

\subsection{Qualitative Analysis}

Within the first month of availability, the research team held three community focus groups to understand the utility of Healthcheck more broadly across diverse populations including outside of the academic ecosystem. In general, participants found the application to function well with good usability. Issues and challenges with the app surrounded differences in personal beliefs and needs in terms of privacy, institutional involvement in personal lives, the right balance between public safety and the need to survive (i.e., make a living, achieve goals), and perceptions about health risks.

We met with a small business focus group (mom and pop stores with foot traffic) $(n=7)$, a medium business focus group (museums, insurers, office buildings, etc.) $(n=6)$ and a faith-based focus group $(n=8)$. Each focus group was 60 minutes and conducted over zoom. Each focus group was recorded for accurate capture of the details. Focus group data was compiled, and thematic analysis was conducted to identify recurring concepts.

Small businesses had the most concern over the use of Healthcheck. Most of the concerns were around employees answering Healthcheck honestly resulting in quarantining for 14 days and leading to shutting down their business. One shop owner got unanimous agreement when he said, "So they complete Healthcheck and then they are told, because of their symptoms, they need to stay home for two weeks. Where does that leave me?" Another concern from the small business group was that they may not answer Healthcheck honestly because if they do not work, they do not get paid. Another business owner said, "My employees don't have two weeks of sick time and they cannot afford to go without pay for two weeks, then what? I bring Healthcheck in and it forces them to lie. No thank you."

Medium sized businesses had strong positive responses toward the utility of Healthcheck for their workers. They felt that using Healthcheck would keep people home when they needed to stay home. One director of human resources said, "So, if they do Healthcheck at home, then we do not need to have 
someone standing at the employee entrance? And we save a person!" Their concern, however, was the need for Healthcheck to sit behind a single sign on authentication system as many did not have a single sign on infrastructure.

Faith-based organizations had a different perspective. At the time of our focus group, they were having someone standing at the door admitting or not admitting parishioners for services. Many commented that this put them in an uncomfortable position. One faith leader said, "If they can know before they come and we do not need to stand at the door telling people they cannot worship today, then this is a great idea." Others were quick to comment though that they did not have the funds to pay for this and while it was a great idea and the utility was sound funds were the limiting factor.

In terms of academic focus groups, we conducted three 60 -minute group interviews with $\mathrm{K}-12$ private schools ( $n=32$ people) as well as institutions of higher ed public $(n=43$ people) and private $(n=12$ people) separately. K-12 focus groups were held at the district level in the way of district board presentations $(n=36$ people). These focus groups were held at staggered times across our deployment starting with higher education and then K-12. All levels of schools saw the immediate utility of Healthcheck. Many commented on the trust level that it was developed by the University with a sound method of development. One element that made the decision easy for schools to agree with the concept and join-in was the support by the Alabama State Governor with CARES Act funds to pay for Healthcheck. One university president succinctly summed up the comments of many, "We can't afford not to do this. Our students and employees are counting on us for protection and instruction. Healthcheck allows for both." Since most academic institutions of all sizes are set up for single sign on (many use Google Schools), implementation was pretty straightforward.

In December 2020, we informally solicited comments from education users. From the 34 schools, we heard from 30 of them. Of the 30 , one small private school said that they could not get a handle on enforcing compliance and have stopped using Healthcheck. The other 29 schools continue to use Healthcheck providing comments such as: "Healthcheck has allowed us to keep everyone on campus and continue with in person instruction." Others, especially from K-12 said, "Once our parents realized that this would keep their children in school and not doing e-learning, compliance was not a problem." A university CIO commented: "Healthcheck has been a no brainer for us. We get our reports and put the data in with some of our data and we get a lot of information. Our COVID team knows what is going on across campus, in the buildings, and in the dorms. We love it."

\section{Discussion}

The COVID-19 symptom assessment and risk assessment tool reported in this manuscript represents a type of tool that has become more commonplace across the globe to mitigate the pandemic. This paper provides a design science research approach to designing and developing these types of tools for wide scale deployment and use. We have included functional requirements, policy driven requirements, and success factors as integral components of the design framework for our artifact. The principles motivating the design of our artifact are grounded in public health, to reduce exposure to COVID-19 while allowing healthcare workers at a major medical university system as well as faculty, staff, and students at educational institutions across one US state to return to work in as safe a manner as possible given the best guidelines available at the time. The Healthcheck tool allowed for COVID-19 symptom checking, risk assessment based on reported symptoms and behavior, and promoted regular use to comply with organizational policies. This was one of the first known solutions that was broadly available to public to accomplish these purposes. It provides a heretofore unavailable avenue for workers and students to return to normal work and school activities in Alabama while trying to balance stakeholder needs to monitor disease fluctuations and user needs for privacy. The design knowledge and design science research framework provided in this study may be useful for developing similar tools in other locations. The software tool provides a design artifact that was tested and evaluated across over 172,000 users and thus provides a complete DSR cycle inclusive of an initial population wide artifact evaluation.

In the evaluation of this artifact, we conducted focus groups to assess usability, and efficacy as a disease mitigation and return-to-work tool. Users complied with use policies more than $62 \%$ of the time, with K-12 schools showing higher compliance than colleges and universities. Using system use data and focus group discussions, findings indicate the system was generally accepted, used regularly, facilitated reduction of disease exposure, and enabled a path back to work and school. While the use statistics provide some value in understanding system uptake and use, we have not yet evaluated statistically significant differences between groups, such as differences in compliance across organizational types, nor behavior and compliance differences between different user groups. This is due to the very early phase of evaluation research in this study and the known difficulties 
determining group differences across use data. Participants from focus groups noted the Healthcheck app was intuitive and useful for accomplishing the goals needed to perform a symptom check and determine risk level. Most issues and challenges revolved around policy and personal value differences reflected in using an app like Healthcheck. Participants found a need to strike a balance between public health needs and personal needs to make a living, continue with personal goal achievement (e.g., education), and for privacy and institutional non-intrusion into their lives. Taken together, the quantitative and qualitative results from this study show promise for such tools for facilitating a difficult policy balance between disease mitigation and continuing to work and study in a normal pattern in life.

\section{Conclusion}

This paper took a design science approach to present the justification, design, development, and early assessment of a return-to-work COVID-19 symptom checker and risk assessor. The system was implemented across 34 institutions of health and education in the US State of Alabama, including over $174 \mathrm{k}$ users with over 4 million total uses between July 2020 and April 2021.

The results of this study indicate the Healthcheck system may provide a strong framework for tools that strike a sound balance in this regard. In terms of broader impacts on ubiquitous healthcare, the artifact in this paper represents further integration of health and healthcare into the daily lives of every individual. Technology enabled remote health checking, risk assessment, and determination for work and school readiness/availability based on individual health or infection status has been represented in this paper and in various settings across the globe as an important set of public health policy activities. Designing such tools that satisfy broad stakeholder groups is an ongoing design challenge.

\section{Acknowledgments}

This work was funded by the State of Alabama through CARES Act funds. The entire team is grateful to Selwyn Vickers for forwarding such an innovative and comprehensive plan, and to The University of Alabama Board of Trustees for their support to keep Alabamians in school and at work during the COVID-19 pandemic. The authors thank Mohanraj Thirumalai and the work of his team in the development of the Healthcheck app as well as Curtis Carver and C. Brian Rivers for their support in the execution of Healthcheck. We also acknowledge the work of our business partner, Craig Parker and his team at Guideway Cares, for their expertise in the seamless scaling and sustaining of Healthcheck. The authors also thank the many participants across Alabama government, schools, universities, and businesses who participated in the requirements gathering interviews. The authors are the only parties responsible for the findings and opinions expressed herein.

\section{References}

[1] Bardhan, I., Chen, H., and Karahanna, E.: 'Connecting systems, data, and people: A multidisciplinary research roadmap for chronic disease management', MIS Quarterly, 2020, 44, (1), pp. 185-200

[2] Sharma, T., and Bashir, M.: 'Use of apps in the COVID-19 response and the loss of privacy protection', Nature medicine, 2020, 26, (8), pp. 1165-1167

[3] Gasser, U., Ienca, M., Scheibner, J., Sleigh, J., and Vayena, E.: 'Digital tools against COVID-19: taxonomy, ethical challenges, and navigation aid', The Lancet Digital Health, 2020

[4] Katzer, R.J.: 'Keeping the Fire House Running: A Proposed Approach to Mitigate Spread of COVID-19 Among Public Safety Personnel', Western Journal of Emergency Medicine, 2020, 21, (3), pp. 546

[5] Hakimi, A.A., and Armstrong, W.B.: 'Hand sanitizer in a pandemic: wrong formulations in the wrong hands', The Journal of Emergency Medicine, 2020, 59, (5), pp. 668-672 [6] Bozdağ, F.: 'The psychological effects of staying home due to the COVID-19 pandemic', The Journal of general psychology, 2020, pp. 1-23

[7] Hevner, A., and Chatterjee, S.: 'Design science research in information systems' (Springer, 2010. 2010)

[8] Peffers, K., Tuunanen, T., Rothenberger, M.A., and Chatterjee, S.: 'A design science research methodology for information systems research', Journal of management information systems, 2007, 24, (3), pp. 45-77

[9] Drechsler, A., and Hevner, A.R.: 'Utilizing, producing, and contributing design knowledge in DSR projects', in Editor (Ed.) $)^{\wedge}$ (Eds.): 'Book Utilizing, producing, and contributing design knowledge in DSR projects' (Springer, 2018, edn.), pp. 82-97

[10] Dalkey, N., and Helmer, O.: 'An experimental application of the Delphi method to the use of experts', Management science, 1963, 9, (3), pp. 458-467

[11] Okoli, C., and Pawlowski, S.D.: 'The Delphi method as a research tool: an example, design considerations and applications', Information \& management, 2004, 42, (1), pp. 15-29 